\title{
Unique Continuation for First Order Systems of PDEs
}

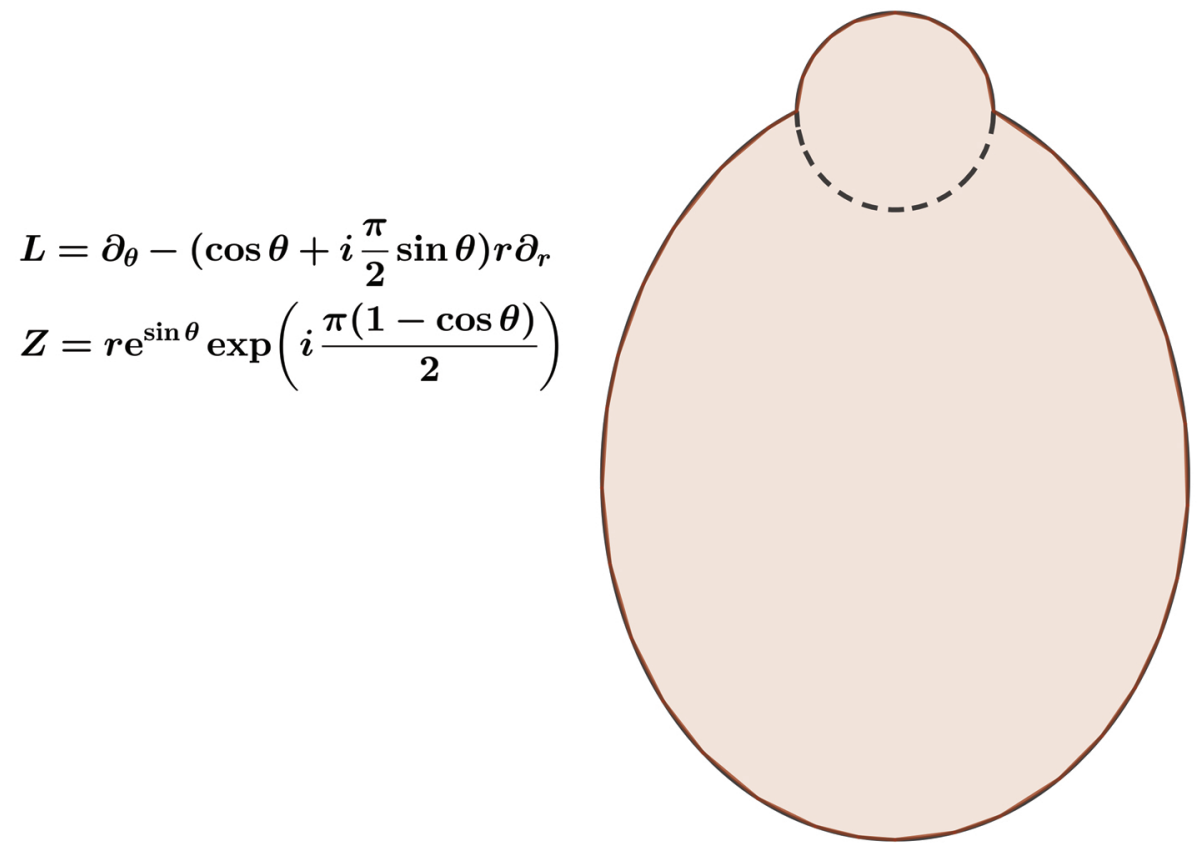

Shiferaw Berhanu

\section{Introduction}

This article is a survey of some of the results on weak and strong unique continuation for systems of linear and nonlinear first order partial differential equations. The linear PDEs arise as sections of a vector subbundle $\mathcal{V}$ of the complexified tangent bundle $\mathbb{C} T M$ of a connected manifold $M$. The bundle $\mathcal{V}$ satisfies the involutivity condition

$$
[\mathcal{V}, \mathcal{V}] \subseteq \mathcal{V}
$$

which means that for any $C^{1}$ sections $X, Y$ of $\mathcal{V}$, the Lie bracket $[X, Y]$ is also a section of $\mathcal{V}$. We will always assume that $\mathcal{V}$ is locally integrable, that is, the orthogonal of $\mathcal{V}$ in the complexified cotangent bundle $\mathbb{C} T^{*} M$ is locally generated by exact forms. In this case, if the fiber dimension of $\mathcal{V}$ over $\mathbb{C}$ is $n$, each point in $M$ has a neighborhood $U$ such that if $\left\{L_{1}, \ldots, L_{n}\right\}$ is a basis of $\mathcal{V}$ over $U$, then there exist

Shiferaw Berhanu is a professor of mathematics at Temple University. His email address is berhanu@temple. edu.

Communicated by Notices Associate Editor Daniela De Silva.

For permission to reprint this article, please contact:

reprint-permission@ams.org.

DOI: https://doi.org/10.1090/noti2351 $m=\operatorname{dim} M-n C^{1}$ sections $Z_{1}, \ldots, Z_{m}$ which are solutions of

$$
L_{j} h=0,1 \leq j \leq n \text {, in } U,
$$

and the differentials $d Z_{1}, \ldots, d Z_{m}$ are linearly independent over $\mathbb{C}$ at each point of $U$. We will call $\left\{Z_{1}, \ldots, Z_{m}\right\}$ a complete set of first integrals on $U$.

When the bundle $\mathcal{V}$ is locally integrable, then it satisfies the involutivity condition (1.1) and we will refer to the pair $(M, \mathcal{V})$ as a locally integrable structure.

In such a structure, given any point $p \in M$, there are local coordinates $x_{1}, \ldots, x_{m}, t_{1}, \ldots, t_{n}$ vanishing at $p$ such that $v$ is generated locally by a basis of the form ( $m$ and $n$ are as before)

$$
L_{j}=\frac{\partial}{\partial t_{j}}+\sum_{k=1}^{m} a_{j k}(x, t) \frac{\partial}{\partial x_{k}}, 1 \leq j \leq n .
$$

For the nonlinear systems

$$
F_{j}\left(x, u, u_{x}\right)=0,1 \leq j \leq n, x=\left(x_{1}, \ldots, x_{N}\right),
$$

that we consider, local coordinates $(x, t) \in \mathbb{R}^{m} \times \mathbb{R}^{n}(m+$ $n=N$ ) can be found in which the equations take the form

$$
u_{t_{j}}=f_{j}\left(x, t, u, u_{x}\right), 1 \leq j \leq n .
$$




\section{The Weak and Strong Unique Continuation Properties}

It will be instructive to consider some examples of locally integrable structures.

Example 2.1. Let $L_{1}, \ldots, L_{n}$ be smooth, linearly independent real vector fields on a domain $\Omega \subseteq \mathbb{R}^{N}$ such that the Lie bracket $\left[L_{i}, L_{j}\right]$ is in the linear span of $L_{1}, \ldots, L_{n}$. Let $\mathcal{V}$ denote the subbundle of $\mathbb{C} T \Omega$ generated by $L_{1}, \ldots, L_{n}$. By the Frobenius theorem, each $p \in \Omega$ is a center of local coordinates $x_{1}, \ldots, x_{N}$ in which the bundle $\mathcal{V}$ is locally generated by $\frac{\partial}{\partial x_{j}}, \quad 1 \leq j \leq n$. Hence $\mathcal{V}$ is locally integrable and, in these coordinates, any solution $u$ has the form $u\left(x_{1}, \ldots, x_{N}\right)=u\left(x_{n+1}, \ldots, x_{N}\right)$.

Example 2.2. Let $\Omega \subseteq \mathbb{C}^{n}$ be a domain, and let $\mathcal{V}$ be the bundle generated by

$$
\frac{\partial}{\partial \bar{z}_{j}}=\frac{1}{2}\left(\frac{\partial}{\partial x_{j}}+\sqrt{-1} \frac{\partial}{\partial y_{j}}\right), 1 \leq j \leq n .
$$

The coordinate functions $z_{j}=x_{j}+\sqrt{-1} y_{j}, 1 \leq j \leq n$, are a complete set of first integrals and so $(\Omega, \mathcal{V})$ is locally integrable. The solutions are holomorphic functions.

Example 2.3. Let $L_{1}, \ldots, L_{n}$ be linearly independent vector fields with real analytic, complex-valued coefficients on a domain $\Omega \subseteq \mathbb{C}^{N}$. Assume that the brackets $\left[L_{i}, L_{j}\right]$ are in the linear span of $L_{1}, \ldots, L_{n}$, and let $\mathcal{V}$ denote the subbundle generated by the $L_{j}$. By the holomorphic version of the Frobenius theorem, $\mathcal{V}$ is locally integrable (see [9]).

Example 2.4 (Embedded CR submanifolds). Let $M \subseteq \mathbb{C}^{n}$ be a smooth real submanifold of dimension $2 n-d$. $M$ is called a CR manifold if for each $p \in M$ there are realvalued, smooth defining functions $\rho_{1}, \ldots, \rho_{d}$ defined on a neighborhood $U$ of $p$ such that the one-forms

$$
\bar{\partial} \rho_{1}, \ldots, \bar{\partial} \rho_{d}
$$

are linearly independent on $M \cap U$. Here, $\bar{\partial} \rho_{j}=$ $\sum_{k=1}^{n} \frac{\partial \rho_{j}}{\partial \bar{z}_{k}} d \bar{z}_{k}$. Let $\mathcal{V}^{\prime}$ be the subbundle of $\mathbb{C} T \mathbb{C}^{n}$ generated by $\frac{\partial}{\partial \bar{z}_{j}}, 1 \leq j \leq n$, and set

$$
\mathcal{V}=\bigcup_{p \in M} \mathcal{V}_{p}(M), \mathcal{V}_{p}(M)=\mathcal{V}_{p}^{\prime} \cap \mathbb{C} T_{p} M .
$$

Since the forms $\bar{\partial} \rho_{j}$ are linearly independent, $\mathcal{V}$ is a subbundle of $\mathbb{C} T M$ of fiber dimension $n-d$. The restrictions of the coordinates $z_{j}=x_{j}+\sqrt{-1} y_{j}(1 \leq j \leq n)$ to $M$ form a complete set of first integrals and so $(M, \mathcal{V})$ is locally integrable.

In general, the forms $\bar{\partial} \rho_{j}$ of locally defining functions may not be linearly independent and so $\mathcal{V}$ may not be a bundle. However, when $M$ is a hypersurface, it is a bundle. When $(M, \mathcal{V})$ is a CR pair, the solutions are called CR functions or CR distributions. Examples of CR functions are provided by the restrictions to $M$ of holomorphic functions. However, CR functions may not even be continuous. For example, if $M$ is the graph

$$
M=\left\{\left(z^{\prime}, s+\sqrt{-1} \varphi\left(z^{\prime}, s\right)\right): z^{\prime} \in \mathbb{C}^{n-1}, s \in \mathbb{R}\right\},
$$

where $\varphi \geq 0$, and smooth, then the function $u\left(z^{\prime}, s\right)=$ $\left(s+\sqrt{-1} \varphi\left(z^{\prime}, s\right)\right)^{1 / 2}$ (with the principal branch of the square root) is a continuous CR function which is not differentiable at the point $\left(z^{\prime}, 0\right)$, where $\varphi\left(z^{\prime}, 0\right)=0$. If $\varphi \equiv 0$, then any function of $s$ is a CR function.

Example 2.5 (Tube structures). Let $x=\left(x_{1}, \ldots, x_{m}\right)$ and $t=\left(t_{1}, \ldots, t_{n}\right)$ denote the coordinates in $\mathbb{R}^{m}$ and $\mathbb{R}^{n}$, respectively. Let $\varphi_{j}(t), 1 \leq j \leq m$, be smooth functions on an open set $U$ and define on $M=\mathbb{R}^{m} \times U$

$$
Z_{j}(x, t)=x_{j}+\sqrt{-1} \varphi_{j}(t), 1 \leq j \leq m .
$$

The differentials $d Z_{1}, \ldots, d Z_{m}$ are linearly independent everywhere on $M$ and their orthogonal in $\mathbb{C} T(M)$ is a bundle $\mathcal{V}$ which has the basis

$$
L_{k}=\frac{\partial}{\partial t_{k}}-\sqrt{-1} \sum_{j=1}^{m} \frac{\partial \varphi_{j}}{\partial t_{k}}(t) \frac{\partial}{\partial x_{j}}, 1 \leq k \leq n .
$$

Thus $(M, \mathcal{V})$ is locally integrable, and it generalizes Example 2.2. Note that when the map $\varphi=\left(\varphi_{1}, \ldots, \varphi_{m}\right): U \rightarrow$ $\mathbb{R}^{m}$ is an immersion, then $(M, \mathcal{V})$ can be identified with a CR submanifold.

A particular case with $m=n=1$ and $\varphi(t)=\frac{t^{2}}{2}$ leads to the planar vector field

$$
L=\frac{\partial}{\partial t}-\sqrt{-1} t \frac{\partial}{\partial x}
$$

which is called the Mizohata vector field and is the simplest nonsolvable partial differential operator in the sense that there exist smooth functions $f$ in a neighborhood of the origin for which the equation $L u=f$ has no solution (not even a distribution solution) in any neighborhood of the origin.

Remark 2.1. There are involutive bundles $\mathcal{V}$ which are not locally integrable. For example, in [19] Nirenberg constructed a vector field in the plane of the form

$$
L=\frac{\partial}{\partial t}-\sqrt{-1}(t+g(x, t)) \frac{\partial}{\partial x}
$$

with $g$ smooth vanishing to infinite order at the origin with the property that for any $C^{1}$ solution $u$ of $L u=0$ near the origin, $d u(0,0)=0$.

Remark 2.2. In the opening graphic which is taken from [18], $L$ defines a vector field in the plane with first integral $Z$ and the picture illustrates the domain of extendability of any solution of $L u=0$ in the unit disc. 
Definition 2.1. The locally integrable structure $(M, \mathcal{V})$ is said to satisfy the weak unique continuation property if any solution that vanishes on a nonempty open subset vanishes on $M$.

Definition 2.2. The locally integrable structure $(M, \mathcal{V})$ satisfies the strong unique continuation property if any solution that is flat at a point vanishes on $M$.

The validity of the weak unique continuation property both for linear and nonlinear systems is connected with the notion of Sussmann's orbits ([20]) which are very useful geometric objects associated with a given family of real vector fields. To describe them briefly, let $X$ be a set of locally defined smooth vector fields on a manifold $M$. Each element of $X$ is defined on some open subset of $M$ and we assume that the union of the domains of the members of $X$ is $M$. We will say two points $p$ and $q$ in $M$ are related if there is a curve $\Gamma:[0, T] \rightarrow M$ with the properties:

(i) $\Gamma(0)=p, \Gamma(T)=q$;

(ii) there exist $t_{0}=0<t_{1}<\cdots<t_{k}=T$ and $X_{i} \in$ $X(1 \leq i \leq k)$ such that for each $i, \Gamma:\left[t_{i-1}, t_{i}\right] \rightarrow M$ is an integral curve of $X_{i}$ or $-X_{i}$.

The equivalence classes of this relation are called the Sussmann orbits of $X$. In [20], it was shown that these orbits can be equipped with a topological and differential structure that makes them submanifolds of $M$.

For a simple example, consider the classical Frobenius foliation which arises from a manifold $M$ and a smooth real subbundle $\mathcal{V}$ of $T M$ which is closed under brackets. In this case, $M$ is a disjoint union of submanifolds each of dimension $k(k=$ the fiber dimension of $\mathcal{V})$ and they are integral manifolds of $\mathcal{V}$. These submanifolds are the Sussmann orbits of the family $X$ that consists of the smooth sections of $\mathcal{V}$. In general, even locally, the dimension of Sussmann's orbits may not be constant. Moreover, the tangent space of an orbit may contain vectors that are not in the Lie algebra generated by the elements of $X$ (see [20], [9]). Thus the concept of Sussmann's orbits is a substantial generalization of the Frobenius foliation.

We can now state a result on the weak unique continuation property for linear systems. The theorem is due to F. Treves ([21]). We will present a proof that appeared in [12].

Theorem 2.1. Let $(M, \mathcal{V})$ be a locally integrable structure, and set

$$
X=\Re(\mathcal{V})=\{S: S=\Re L, L \text { a smooth section of } \mathcal{V}\} .
$$

If a solution $u$ on $M$ vanishes in a neighborhood of $p$, then it vanishes in a neighborhood of the Sussmann orbit of $X$ through $p$. Thus the support of $u$ is a union of orbits of $X$. In particular, if $M$ is an orbit, then $(M, \mathcal{V})$ satisfies the weak unique continuation property.
Example 2.6. Let $(M, \mathcal{V})$ be locally integrable and $X=$ $\Re(\mathcal{V})$. Suppose at each $p \in M$, the linear span of all of the repeated brackets of sections of $X$ equals $T_{p} M$. Then $M$ is the only orbit of $X$ and so by Theorem 2.1 , the weak unique continuation property holds for $(M, \mathcal{V})$.

Remark 2.3. There are examples of locally integrable structures where $M$ is the only orbit of $X=\Re(\mathcal{V})$ although the hypothesis in Example 2.6 is not satisfied.

Example 2.7. In the work [14] P. Cohen gave an example of a smooth vector field

$$
L=\frac{\partial}{\partial y}+a(x, y) \frac{\partial}{\partial x}
$$

in the plane with a smooth solution $u$ on $\mathbb{R}^{2}$ of $L u=0$ whose support $=\{(x, y): y \geq 0\}$. The support of such a solution is not a union of the orbits of $X=\{\Re L, \mathfrak{I} L\}$. This example shows that Theorem 2.1 may not hold for involutive structures that are not locally integrable.

\section{Proof of Theorem 2.1}

The proof of Theorem 2.1 will use the uniqueness in the noncharacteristic Cauchy problem for locally integrable structures which in turn is a consequence of the celebrated approximation theorem of Baouendi and Treves ([5]). Given a locally integrable structure $(M, \mathcal{V})$ and a complete set of first integrals $Z=\left(Z_{1}, \ldots, Z_{m}\right)$ on an open subset $U$, if $F\left(z_{1}, \ldots, z_{m}\right)$ is a holomorphic function on a neighborhood of $Z(U)$, then the function $F\left(Z_{1}, \ldots, Z_{m}\right)$ is a solution on $U$. In general, even locally, solutions may not have such a form. The approximation theorem states that any solution in a locally integrable structure $(M, \mathcal{V})$ can be locally approximated by solutions $F\left(Z_{1}, \ldots, Z_{m}\right)$ of the above form. Moreover, the theorem shows that a solution is determined by its restriction to certain $m$-dimensional $\left(\operatorname{dim}_{\mathbb{C}} \mathcal{V}=n, m=\operatorname{dim} M-n\right)$ submanifolds called maximally real submanifolds. These submanifolds are generalizations of the totally real submanifolds of $\mathbb{C}^{n}$ with maximal dimension.

Definition 3.1. Let $(M, \mathcal{V})$ be a locally integrable structure, $\operatorname{dim}_{\mathbb{C}} \mathcal{V}=n, m=\operatorname{dim} M-n$. A submanifold $S \subset M$ is called maximally real if for each $p \in S$,

$$
\mathbb{C} T_{p} S \oplus \mathcal{V}_{p}=\mathbb{C} T_{p} M .
$$

Locally, there is a good and useful description of such submanifolds. Observe first that a submanifold $S$ is maximally real if and only if for any complete set of first integrals $Z_{1}, \ldots, Z_{m}$ on a neighborhood $U$ of $p$ in $S$, the restrictions of the $Z_{j}$ to $U \cap S$ have linearly independent differentials. If $U$ is small enough, we can find local coordinates $x_{1}, \ldots, x_{m}, t_{1}, \ldots, t_{n}$ that vanish at $p$ and a complete set of first integrals $Z_{1}, \ldots, Z_{m}$ such that

$$
Z_{j}(x, t)=x_{j}+\sqrt{-1} \varphi_{j}(x, t), \varphi_{j}(0,0)=0,
$$




$$
d_{x} \varphi_{j}(0,0)=0,1 \leq j \leq m,
$$

and

$$
U \cap S=\{(x, t) \in U: t=0\} .
$$

The approximation theorem of Baouendi and Treves can be stated as follows.

Theorem 3.1 ([5]). Let $(\Omega, \mathcal{V})$ be a locally integrable structure where $\Omega \subset \mathbb{R}^{m+n}$ is open, and let $Z_{1}, \ldots, Z_{m}$ be a complete set of first integrals near a point $p \in \Omega$. Then there exists $a$ neighborhood $U$ of $p$ such that if $u$ is any continuous solution, there exists a sequence of entire functions $H_{k}\left(z_{1}, \ldots, z_{m}\right)$ that satisfy

$$
u(x)=\lim _{k \rightarrow \infty} H_{k}(Z(x)) \text { uniformly on } U .
$$

Remark 3.1. The convergence in the theorem also holds for functions or distributions in various function spaces (see [9]).

The entire functions $H_{k}$ in the theorem have an explicit form. Given $p \in \Omega$, let $S$ be a maximally real submanifold through $p$. Choose local coordinates $x_{1}, \ldots, x_{m}, t_{1}, \ldots, t_{n}$ vanishing at $p$ on a neighborhood of $p$ such that

$$
\begin{gathered}
U \cap S=\{(x, t) \in U: t=0\}, \\
\text { and } Z_{j}(x, t)=x_{j}+\sqrt{-1} \varphi_{j}(x, t), 1 \leq j \leq m,
\end{gathered}
$$

is a complete set of first integrals on $U$, with $\varphi_{j}$ real-valued, $\varphi_{j}(0,0)=0, d_{x} \varphi_{j}(0,0)=0$.

We may assume that $U=\left\{(x, t):|x| \leq \frac{r}{4},|t| \leq d\right\}$. Let $g(x) \in C_{0}^{\infty}\left(\mathbb{R}^{m}\right)$ with $g(x) \equiv 1$ for $|x| \leq \frac{3 r}{4}$ and $g(x) \equiv 0$ for $|x| \geq r$.

For $k=1,2, \ldots$, define

$$
H_{k}(z)=\left(\frac{k}{\pi}\right)^{\frac{m}{2}} \int_{\mathbb{R}^{m}} e^{-k \sum_{j=1}^{m}\left(z_{j}-Z_{j}\left(x^{\prime}, 0\right)\right)^{2}} g\left(x^{\prime}\right) u\left(x^{\prime}, 0\right) d_{x^{\prime}} Z,
$$

where $d_{x^{\prime}} Z=\operatorname{det} Z_{x^{\prime}}\left(x^{\prime}, 0\right) d x^{\prime}$. Theorem 3.1 is proved by showing that $H_{k}(Z(x, t))$ converges uniformly to $u(x, t)$ on $U$ for $r, d$ small enough, but independent of $u$.

Theorem 3.1 has the following immediate consequences.

Corollary 3.1. Let $(M, \mathcal{V})$ be a locally integrable structure and $S \subseteq M$ a maximally real submanifold. If $u$ is a continuous solution on $M$ and vanishes on $S$, then it vanishes on a neighborhood of $S$.

Corollary 3.2. Let $(M, \mathcal{V})$ be a locally integrable structure and $Y \subseteq M$ a noncharacteristic hypersurface in the sense that at each point $p \in Y, \mathcal{V}_{p} \subsetneq \mathbb{C} T_{p} Y$. If $u$ is a continuous solution on $M$ and it vanishes on $Y$, then it vanishes on a neighborhood of $Y$.

Corollary 3.2 is proved using Corollary 3.1 and the fact that a noncharacteristic hypersurface contains a maximally real submanifold through each of its points.
We are now ready to present a proof of Theorem 2.1 from [12]. We will use some concepts and a result of Bony ([13]).

Definition 3.2. Let $\Omega \subseteq \mathbb{R}^{n}$ be an open set and $F \subseteq \Omega$ a closed subset. A vector $v$ is said to be normal to $F$ at $x_{0}$ in $F$ if there is an open ball $B \subseteq \Omega \backslash F$ centered at $x$ such that $x_{0} \in \partial B$ and $v=\lambda\left(x-x_{0}\right)$ for some $\lambda>0$.

Definition 3.3. Suppose $\Omega$ and $F$ are as in Definition 3.2. A vector field $X(x)$ is said to be tangent to $F$ if whenever $v$ is normal to $F$ at $x_{0} \in F$, the vector $X\left(x_{0}\right)$ is orthogonal to $v$.

Bony proved the following.

Theorem 3.2 ([13]). Suppose $\Omega \subseteq \mathbb{R}^{n}$ is an open set and $F \subseteq \Omega$ a closed subset. Let $X(x)$ be a Lipschitz vector field in $\Omega$ which is tangent to $F$. If an integral curve of $X$ intersects $F$ at a point, then it is entirely contained in $F$.

Let $(M, \mathcal{V}), X$ be as in the statement of Theorem 2.1. Suppose $u$ is a solution on $M$ and $F=$ the support of $u$. Let $\Omega=M \backslash F$. Define $N(F)$ to be the set of $v \in$ $T^{*} M \backslash 0$ over points in $F$ such that there exists a realvalued, smooth function $f$ defined near $p=\pi(v)$ satisfying $f(p)=0, d f(p)=v$, and $f \leq 0$ on $F$ near $p$. Fix $p \in F$ and suppose $v \in N(F)$ with $\pi(v)=p$. Let $f$ be as above with $d f(p)=v$. Since $v \neq 0$, the zero set of $f$ is a smooth hypersurface near $p$. Observe that $u=0$ on a side of this hypersurface. Since $p \in F$, by Corollary $3.2,\{f=0\}$ has to be characteristic to $\mathcal{V}$ at $p$. Thus for any Lipschitz section $Y$ of $\Re(\mathcal{V}),\langle v, Y(p)\rangle=0$. By Theorem 3.2, the integral curve of $Y$ through $p$ has to lie in $F$.

\section{The Strong Unique Continuation Property}

There are some classes of locally integrable structures where the validity of the strong unique continuation is well understood. If $(M, \mathcal{V})$ is a smooth locally integrable structure where the dimension of $M$ is two and $\mathcal{V}$ is one dimensional, then the strong unique continuation holds if and only if every local first integral $Z$ is an open mapping into the complex plane. If $(M, \mathcal{V})$ is a tube structure as in Example 2.5, and the map $\varphi(t)$ is real analytic, then the strong unique continuation property holds if and only if for every $\xi \in \mathbb{R}^{m}, \xi \neq 0$, the function $t \mapsto \varphi(t) \cdot \xi$ does not have a local extremum. This is a consequence of the fact that this latter condition is equivalent to the analyticity of all solutions (see [6]).

In the rest of this section we focus on CR vector fields and present some of the known results on the strong unique continuation property for CR functions.

If $f$ is a continuous function on the closure of a domain $\Omega \subseteq \mathbb{R}^{n}$, we will say it is flat at $p \in \partial \Omega$ if

$$
f(x)=o\left(|x-p|^{N}\right) \text { for all } N=1,2, \ldots .
$$


We begin with the case of holomorphic functions of one variable since boundary unique continuation results for such functions have important applications to the unique continuation problem for CR functions.

Let $\Delta^{+}=\{z \in \mathbb{C}:|z|<1, \mathfrak{\Im} z>0\}$ be the half ball in the plane. The following result provides a sufficient condition for the boundary unique continuation property to hold for holomorphic functions on $\Delta^{+}$.

Theorem 4.1 ([16]). Let $f$ be a holomorphic function on $\Delta^{+}$, continuous up to $(-1,1) \subseteq \partial \Delta^{+}$. Assume that the real part of $f$ is nonnegative on $(-1,1)$. If $f$ is flat at 0 , then $f \equiv 0$.

In [1] H. Alexander proved a significant generalization of the preceding theorem: if $f$ is holomorphic on $\Delta^{+}$, continuous up to $(-1,1)$, and $f(-1,1)$ is a nonspiraling set, then $f \equiv 0$ whenever it is flat at 0 . A good example of a nonspiraling set is the complement in $\mathbb{C}$ of a curve emanating from the origin. For other results along this line, see [10].

Many well-known results on unique continuation for CR mappings follow from the one variable results described above. We will present next some of these results and indicate how they can be proved.

Let $M \subseteq \mathbb{C}^{n}$ be a totally real submanifold of class $C^{1, \alpha}(0<\alpha<1)$ of dimension $n, 0 \in M$. We may assume that in a neighborhood $U$ of the origin in $\mathbb{C}^{n}$, $M=\left\{z \in \mathbb{C}^{n}: \mathfrak{I} z=\varphi(\Re z)\right\}, \varphi \in C^{1, \alpha}(U), \varphi$ real-valued, $\varphi(0)=0, \varphi^{\prime}(0)=0$.

A wedge $\mathcal{W}$ with edge $M$ is a set of the form

$$
\mathcal{W}=\{w \in U: \mathfrak{I} w-\varphi(\Re w) \in \Gamma\},
$$

where $\Gamma \subseteq \mathbb{R}^{n}$ is a convex open cone.

Theorem 4.2 ([2]). Let $M$ and $\mathcal{W}$ be as above, with $M$ of class $C^{2}$ and $h: \overline{\mathcal{W}} \rightarrow \mathbb{C}^{k}$ continuous, holomorphic on $\mathcal{W}$ satisfying:

(1) $h$ is flat at 0 , and $C^{2}$.

(2) $h(M) \subseteq M^{\prime}$ with $M^{\prime}$ a totally real submanifold of class

Then $h \equiv 0$ in the connected component of 0 in $\overline{\mathcal{W}}$.

Observe that if $M$ and $M^{\prime}$ are real analytic, Theorem 4.2 follows from the classical edge of the wedge theorem.

In [16] the authors defined a hypersurface $M \subseteq \mathbb{C}^{n}$ to be positive at a point $p \in M$ if there is a holomorphic change of coordinates mapping $p$ to 0 and in the new coordinates $\left(z^{\prime}, z_{n}\right)=\left(z^{\prime}, x_{n}+\sqrt{-1} y_{n}\right) \in \mathbb{C}^{n-1} \times \mathbb{C}, M$ is given by $\left\{y_{n}=\rho\left(z^{\prime}, x_{n}, y_{n}\right)\right\}$ with $\rho(0)=0, d \rho(0)=0$, and $\rho\left(z^{\prime}, x_{n}+\sqrt{-1} y_{n}\right)>0$ when $z^{\prime} \neq 0$. When $M$ is positive at $p$, there is a complex hypersurface through $p$ that intersects $M$ locally only at $p$. They proved a version of the following theorem under such assumption.

Theorem 4.3 ([10]). Let $M_{1} \subseteq \mathbb{C}^{n+d}$ be a smooth CR manifold of CR dimension $n$ and $M_{2} \subseteq \mathbb{C}^{m}$ a hypersurface. Suppose $f: M_{1} \rightarrow M_{2}$ is a continuous CR mapping which extends to a holomorphic mapping into a wedge $\mathcal{W}$ with edge $M_{1}$ at $0 \in M_{1}$. Suppose there is a complex hypersurface that locally intersects $M_{2}$ only at 0 . If $f$ is flat at 0 , then $f \equiv 0$.

Theorems 4.2 and 4.3 can be proved using the one variable result of Theorem 4.1 and a result on the existence of partially attached analytic discs (Theorem 7.4.12 in [3]).

Theorem 4.1 may be considered as a boundary unique continuation result for harmonic functions on a half-disc in the plane. It has been extended to harmonic functions in higher dimensions in [4], and to solutions of more general equations in [11], [7], and [8].

\section{Nonlinear Systems}

Let $F_{1}\left(x, \zeta_{0}, \zeta\right), \ldots, F_{n}\left(x, \zeta_{0}, \zeta\right)$ be smooth functions of $\left(x, \zeta_{0}, \zeta\right) \in U \times \mathbb{C} \times \mathbb{C}^{N}$, where $U \subseteq \mathbb{R}^{N}$ is open, $x=$ $\left(x_{1}, \ldots, x_{N}\right), \zeta_{0} \in \mathbb{C}, \zeta=\left(\zeta_{1}, \ldots, \zeta_{N}\right) \in \mathbb{C}^{N}$. We assume that the $F_{j}$ are holomorphic in $\left(\zeta_{0}, \zeta\right)$. We will be considering nonlinear systems of first order PDEs of the form

$$
F_{j}\left(x, u(x), u_{x}(x)\right)=0,1 \leq j \leq n,
$$

that generalize the linear systems (1.2).

The linear independence of system (1.2) is generalized by assuming that the wedge product

$$
d_{\zeta} F_{1} \wedge \cdots \wedge d_{\zeta} F_{n} \neq 0 .
$$

Here $d_{\zeta}$ is the exterior derivative in $\zeta=\left(\zeta_{1}, \ldots, \zeta_{N}\right)$. It follows that $n \leq N$. Condition (5.2) implies that if

$$
\Sigma=\left\{\left(x, \zeta_{0}, \zeta\right): F_{j}\left(x, \zeta_{0}, \zeta\right)=0,1 \leq j \leq n\right\}
$$

is nonempty, then it is a smooth manifold.

The involutivity of the linear system (1.2) is generalized as follows: given $F=F\left(x, \zeta_{0}, \zeta\right)$ smooth, holomorphic in $\left(\zeta_{0}, \zeta\right)$, define the holomorphic Hamiltonian of $F$ by

$$
\begin{aligned}
H_{F}= & \sum_{i=1}^{N} \frac{\partial F}{\partial \zeta_{i}} \frac{\partial}{\partial x_{i}}-\sum_{i=1}^{N}\left(\frac{\partial F}{\partial x_{i}}+\zeta_{i} \frac{\partial F}{\partial \zeta_{0}}\right) \frac{\partial}{\partial \zeta_{i}} \\
& +\left(\sum_{i=1}^{N} \zeta_{i} \frac{\partial F}{\partial \zeta_{i}}-F\right) \frac{\partial}{\partial \zeta_{0}}+\frac{\partial F}{\partial \zeta_{0}} .
\end{aligned}
$$

If $G=G\left(x, \zeta_{0}, \zeta\right)$ is also a similar function, we define the Poisson bracket $\{F, G\}$ by

$$
\{F, G\}=H_{F} G .
$$

We will assume that the system (5.1) satisfies the involutivity condition

$$
\left\{F_{j}, F_{k}\right\}=0 \text { on } \Sigma \text { for } j, k=1, \ldots, n .
$$

Condition (5.6) is a formal integrability condition for system (5.1). Indeed, if $u$ is a $C^{2}$ solution of the system, it can be shown that $\left\{F_{j}, F_{k}\right\}=0$ on $\left\{\left(x, u(x), u_{x}(x)\right)\right\}$ for all $1 \leq j, k \leq n$. 
With a solution $u$ of (5.1), we associate the linear vector fields

$$
L_{j}^{u}=\sum_{k=1}^{N} \frac{\partial F_{j}}{\partial \zeta_{k}}\left(x, u(x), u_{x}(x)\right) \frac{\partial}{\partial x_{k}}, 1 \leq j \leq n .
$$

These vector fields are called the linearized operators of system (5.1) at the solution $u$. If for $v \in C^{1}(U)$ we define $F_{j}^{v}$ by

$$
F_{j}^{v}(x)=F_{j}\left(x, v(x), v_{x}(x)\right),
$$

then $L_{j}^{u}(v)$ is the principal part of the Fréchet derivative of the map

$$
v \mapsto F_{j}^{v}
$$

at $u$. Condition (5.2) guarantees that $L_{1}^{u}, \ldots, L_{n}^{u}$ are linearly independent and span a bundle over $U$ which will be denoted by $\mathcal{V}^{u}$. The bundle $\mathcal{V}^{u}$ is involutive. In [21], the following result on unique continuation is proved.

Theorem 5.1. Let $u$ and $v$ be two $C^{2}$ functions on an open set $\Omega \subseteq \mathbb{R}^{N}$ that are solutions of the system of equations

$$
F_{j}\left(x, w(x), w_{x}(x)\right)=0,1 \leq j \leq n,
$$

where the $F_{j}\left(x, \zeta_{0}, \zeta\right)$ are real analytic, holomorphic in $\left(\zeta_{0}, \zeta\right)$.

Let

$$
X=\mathfrak{R}\left(\mathcal{V}^{u}\right)=\left\{S: S=\Re L, L \text { a } C^{1} \text { section of } \mathcal{V}^{u}\right\} .
$$

Assume that the $F_{j}$ satisfy (5.2) and (5.6).

If $u=v$ in a neighborhood of a point $p \in \Omega$, then $u=v$ in a neighborhood of the Sussmann orbit of $X$ through $p$.

We mention that a proof can be given using Bony's result as in the linear case together with the uniqueness theorem of Métivier ([17]) in the Cauchy problem for real analytic, noncharacteristic hypersurfaces.

\section{Concluding Remarks}

Compared with the weak unique continuation property, the question of when the strong unique continuation property is valid for systems of complex vector fields is much less understood. We mention here the following wellknown conjecture due to Baouendi-Rothschild and Huang ([15]) which remains open.

Conjecture 6.1. Let $M_{1}$ and $M_{2}$ be connected real hypersurfaces in $\mathbb{C}^{n}$ with $n \geq 2$. Let $f$ be a smooth map from $M_{1}$ to $M_{2}$ that extends to a holomorphic map on a side of $M_{1}$. Assume that both $M_{1}$ and $M_{2}$ are of finite D'Angelo type and $f$ is flat at $p_{0} \in M_{1}$ in the sense that $\left|f(p)-f\left(p_{0}\right)\right| \leq C_{N}\left|p-p_{0}\right|^{N}$ for each $N$, where $C_{N}$ is a constant depending on $N$. Then $f$ is a constant map.

Let $M$ be a smooth real hypersurface of $\mathbb{C}^{n}, p \in M$, and $r$ a defining function for $M$. The $\mathrm{D}^{\prime}$ Angelo type of $M$ at $p$ is defined by $\Delta(M, p)=\sup _{\gamma \in \mathcal{C}} \frac{\nu(r \circ \gamma)}{\nu \circ \gamma}$, where $\mathcal{C}$ is the set of nonconstant holomorphic germs $\gamma$ at $0 \in \mathbb{C}$ with $\gamma(0)=p$ and $\nu(r \circ \gamma)$ denotes the order of vanishing of the function $r \circ \gamma$ at 0 . A point $p$ is said to be of finite D'Angelo type if $\Delta(M, p)$ is finite.

ACKNOWLEDGMENTS. The author is grateful to A. Meziani for supplying the graphic from [18], and to $\mathrm{X}$. Huang and the referee for their helpful comments. His research is supported in part by NSF DMS grant 1855737.

\section{References}

[1] H. Alexander, A weak Hopf lemma for holomorphic mappings, Indag. Math. (N.S.) 6 (1995), no. 1, 1-5, DOI 10.1016/0019-3577(95)98196-1. MR1324433

[2] Serge Alinhac, M. S. Baouendi, and Linda Preiss Rothschild, Unique continuation and regularity at the boundary for holomorphic functions, Duke Math. J. 61 (1990), no. 2, 635653, DOI 10.1215/S0012-7094-90-06123-X. MR1074309

[3] M. Salah Baouendi, Peter Ebenfelt, and Linda Preiss Rothschild, Real submanifolds in complex space and their mappings, Princeton Mathematical Series, vol. 47, Princeton University Press, Princeton, NJ, 1999, DOI 10.1515/9781400883967. MR1668103

[4] M. S. Baouendi and Linda Preiss Rothschild, A local Hopf lemma and unique continuation for harmonic functions, Internat. Math. Res. Notices 8 (1993), 245-251, DOI 10.1155/S1073792893000273. MR1233452

[5] M. S. Baouendi and F. Trèves, A property of the functions and distributions annihilated by a locally integrable system of complex vector fields, Ann. of Math. (2) 113 (1981), no. 2, 387-421, DOI 10.2307/2006990 MR607899

[6] M. S. Baouendi and F. Trèves, A microlocal version of Bochner's tube theorem, Indiana Univ. Math. J. 31 (1982), no. $6, \quad 885-895$, DOI 10.1512/iumj.1982.31.31060 MR674873

[7] S. Berhanu, Boundary unique continuation for a class of elliptic equations, Amer. J. Math., to appear.

[8] S. Berhanu, Boundary unique continuation for the Laplace equation and the biharmonic operator, Comm. Anal. Geom., to appear.

[9] Shiferaw Berhanu, Paulo D. Cordaro, and Jorge Hounie, An introduction to involutive structures, New Mathematical Monographs, vol. 6, Cambridge University Press, Cambridge, 2008, DOI 10.1017/CBO9780511543067. MR2397326

[10] S. Berhanu and Jorge Hounie, A Hopf lemma for holomorphic functions in Hardy spaces and applications to CR mappings, J. Anal. Math. 138 (2019), no. 2, 835-855, DOI 10.1007/s11854-019-0047-1 MR3996059

[11] S. Berhanu and J. Hounie, A local Hopf lemma and unique continuation for the Helmholtz equation, Comm. Partial Differential Equations 43 (2018), no. 3, 448-466, DOI 10.1080/03605302.2018.1446165 MR3804204

[12] S. Berhanu and G. A. Mendoza, Orbits and global unique continuation for systems of vector fields, J. Geom. Anal. 7 (1997), no. 2, 173-194, DOI 10.1007/BF02921719. MR1646756 
[13] Jean-Michel Bony, Principe du maximum, inégalite de Harnack et unicité du problème de Cauchy pour les opérateurs elliptiques dégénérés (French, with English summary), Ann. Inst. Fourier (Grenoble) 19 (1969), no. fasc. 1, 277-304 xii. MR262881

[14] P. Cohen, The non-uniqueness of the Cauchy problem, O.N.R, Tech. Report 93, Stanford, 1990.

[15] Xiaojun Huang, On some problems in several complex variables and CR geometry, First International Congress of Chinese Mathematicians (Beijing, 1998), AMS/IP Stud. Adv. Math., vol. 20, Amer. Math. Soc., Providence, RI, 2001, pp. 383-396. MR1830195

[16] Xiaojun Huang and Steven G. Krantz, A unique continuation problem for holomorphic mappings, Comm. Partial Differential Equations 18 (1993), no. 1-2, 241-263, DOI 10.1080/03605309308820929 MR1211733

[17] Guy Métivier, Uniqueness and approximation of solutions of first order nonlinear equations, Invent. Math. 82 (1985), no. 2, 263-282, DOI 10.1007/BF01388803. MR809715

[18] Abdelhamid Meziani, Solvability of planar complex vector fields with homogeneous singularities, Complex Var. Elliptic Equ. 62 (2017), no. 10, 1447-1473, DOI 10.1080/17476933.2017.1279611. MR3677947

[19] L. Nirenberg, On a question of Hans Lewy, Russian Math. Surveys 29 (1974), 251-262. MR492752

[20] Héctor J. Sussmann, Orbits of families of vector fields and integrability of distributions, Trans. Amer. Math. Soc. 180 (1973), 171-188, DOI 10.2307/1996660. MR321133

[21] François Trèves, Hypo-analytic structures: Local theory, Princeton Mathematical Series, vol. 40, Princeton University Press, Princeton, NJ, 1992. MR1200459

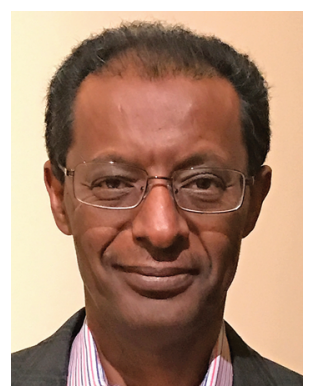

Shiferaw Berhanu

\section{Credits}

Opening graphic is courtesy of A. Meziani.

Author photo is courtesy of the author.

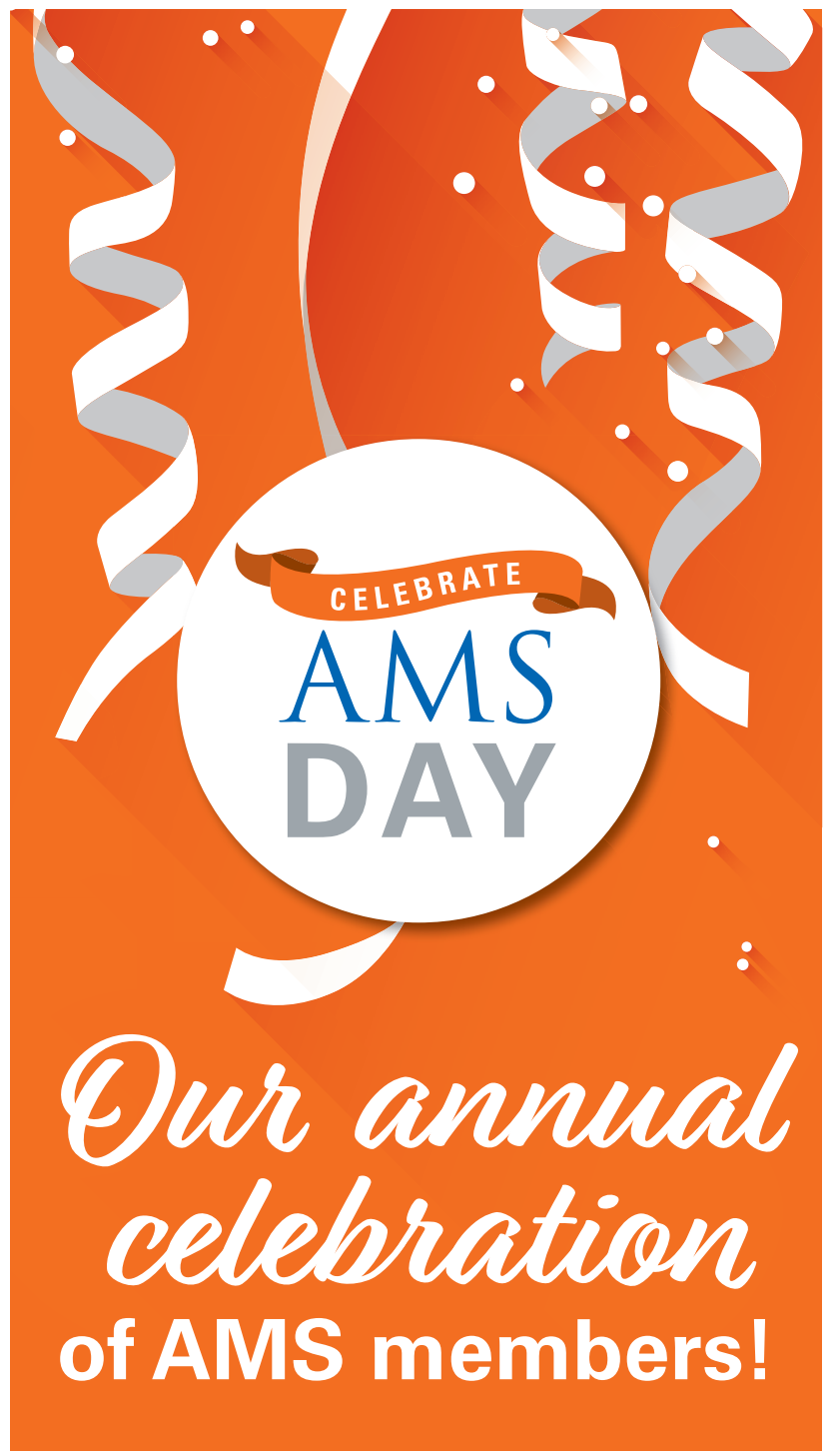

Join us on Monday, November 29 ${ }^{\text {th }}$ as we honor our AMS members via "AMS Day," a day of specials on AMS publications, membership, and much more! Stay tuned on social media and membership communications for details about this exciting day.

\section{Spread the word about \#AMSDay today!}

